\title{
A note on hyperbolic quaternions
}

\author{
Işıl Arda Kösal ${ }^{\mathrm{a}^{*}}$ \\ ${ }^{a}$ Department of Mathematics, Faculty of Arts and Sciences, Sakarya University, Sakarya, Turkey \\ ${ }^{*}$ Corresponding author E-mail: isil.arda@ogr.sakarya.edu.tr
}

\section{Article Info}

Keywords: Hyperbolic quaternion, Euler's formula, De Moivre's formula 2010 AMS: 11R52, $20 G 20$

Received: 18 January 2018

Accepted: 28 February 2018

Available online: 30 September 2018

\begin{abstract}
In this work, we introduce hyperbolic quaternions and their algebraic properties. Moreover, we express Euler's and De Moivre's formulas for hyperbolic quaternions.
\end{abstract}

\section{Introduction}

Real quaternions were introduced by Hamilton (1805-1865) in 1843 as he looked for ways of extending complex numbers to higher spatial dimensions. So the set of real quaternions can be represented as [1]

$$
H=\left\{q=a_{0}+a_{1} i+a_{2} j+a_{3} k: a_{0}, a_{1}, a_{2}, a_{3} \in R \text { and } i, j, k \notin R\right\},
$$

where

$$
i^{2}=j^{2}=k^{2}=-1, i j=k-j i, j k=i=-k j, k i=j=-i k .
$$

From these ruled it follows immediately that multiplication of real quaternions is not commutative. The roots of a real quaternions were given by Niven [7] and Brand [8] proved De Moivre's theorem and used it to find $n t h$ roots of a real quaternion. Cho [2] generalized Euler's formula and De Moivre's formula for real quaternions. Also, he showed that there are uncountably many unit quaternions satisfying $x^{n}=1$ for $n \geq 3$. Using De Moivre's formula to find roots of real quaternion is more useful way.

After the discovery of real quaternions by Hamilton, MacFarlane [4] in 1981 introduced the set of hyperbolic quaternions. The hyperbolic quaternions are not commutative like real quaternions. But the set of hyperbolic quaternions contains zero divisors [6].

In this work, we express Euler and De moivre's formulas for hyperbolic quaternions after we give some algebraic properties of hyperbolic quaternions.

\section{Hyperbolic Quaternions}

A set of hyperbolic quaternions are denoted by

$$
K=\left\{q=a_{0}+a_{1} i+a_{2} j+a_{3} k: a_{0}, a_{1}, a_{2}, a_{3} \in R \text { and } i, j, k \notin R\right\}
$$

where

$$
i^{2}=j^{2}=k^{2}=1, i j=k-j i, j k=i=-k j, k i=j=-i k .
$$

A hyperbolic quaternion $q=a_{0}+a_{1} i+a_{2} j+a_{3} k$ is pieced into two parts with scalar piece $S(q)=a_{0}$ and vectorial piece $\vec{V}(q)=$ $a_{1} i+a_{2} j+a_{3} k$. We also write $q=S(q)+\vec{V}(q)$. Let a hyperbolic quaternion be $q_{n}=a_{n}+a_{n} i+a_{n} j+a_{n} k$ for $n=0,1$. Addition and subtraction of a hyperbolic quaternions is defined by

$$
\begin{array}{r}
q_{0} \pm q_{1}=\left(a_{0}+b_{0} i+c_{0} j+d_{0} k\right) \pm\left(a_{1}+b_{1} i+c_{1} j+d_{1} k\right) \\
=\left(a_{0} \pm a_{1}\right)+\left(b_{0} \pm b_{1}\right) i+\left(c_{0} \pm c_{1}\right) j+\left(d_{0} \pm d_{1}\right) k .
\end{array}
$$


Scalar multiplication of a hyperbolic quaternion is defined by

$$
\lambda q=\lambda(a+b i+c j+d k)=\lambda a+\lambda b i+\lambda c j+\lambda d k
$$

for any $\lambda \in R$. Then, the set $K$ is a vector space over $R$. Multiplication of hyperbolic quaternions are defined by

$$
\begin{aligned}
& q_{0} q_{1}=\left(a_{0}+b_{0} i+c_{0} j+d_{0} k\right)\left(a_{1}+b_{1} i+c_{1} j+d_{1} k\right) \\
& \quad=\left(a_{0} a_{1}+b_{0} b_{1}+c_{0} c_{1}+d_{0} d_{1}\right)+\left(a_{0} b_{1}+b_{0} a_{1}+c_{0} d_{1}-d_{0} c_{1}\right) i \\
& \quad+\left(a_{0} c_{1}-b_{0} d_{1}+c_{0} a_{1}+d_{0} b_{1}\right) j+\left(a_{0} d_{1}+b_{0} c_{1}-c_{0} b_{1}+d_{0} a_{1}\right) k
\end{aligned}
$$

Equation (2.5) can be represented by means of matrix multiplication. The representation as a $4 \times 4$ real matrix is

$$
\left(\begin{array}{cccc}
a_{0} & b_{0} & c_{0} & d_{0} \\
b_{0} & a_{0} & -d_{0} & c_{0} \\
c_{0} & d_{0} & a_{0} & -b_{0} \\
d_{0} & -c_{0} & b_{0} & a_{0}
\end{array}\right)
$$

which is a useful way to compute quaternion multiplication

$$
\left(\begin{array}{l}
r_{0} \\
r_{1} \\
r_{2} \\
r_{3}
\end{array}\right)=\left(\begin{array}{cccc}
a_{0} & b_{0} & c_{0} & d_{0} \\
b_{0} & a_{0} & -d_{0} & c_{0} \\
c_{0} & d_{0} & a_{0} & -b_{0} \\
d_{0} & -c_{0} & b_{0} & a_{0}
\end{array}\right)\left(\begin{array}{l}
a_{1} \\
b_{1} \\
c_{1} \\
d_{1}
\end{array}\right)
$$

where $p q=r_{0}+r_{1} i+r_{2} j+r_{3} k$.

Unlike the real quaternions, the hyperbolic quaternion is not associative due to $(i j) j \neq i(j j)$. Moreover, it is not commutative. The conjugate of a hyperbolic quaternion is defined by

$$
\bar{q}=a-b i-c j-d k .
$$

The conjugate of hyperbolic quaternions satisfies the properties $\overline{(\bar{p})}=p$ and $\overline{(p q)}=\bar{q} \bar{p}$ for $p, q \in K$.

The scalar and vector parts of $q \in K$ are defined

$$
S(q)=\frac{1}{2}(q+\bar{q}), \quad \vec{V}(q)=\frac{1}{2}(q-\bar{q}) .
$$

We note that

$$
\begin{aligned}
& S(\bar{q})=\frac{1}{2}(\bar{q}+q)=S(q), \\
& S(p+q)=S(p)+S(q) .
\end{aligned}
$$

Let $p$ and $q$ be hyperbolic quaternions. Then, inner product of them is defined to be the real number

$$
\langle p, q\rangle=S(p \bar{q}) .
$$

As is easily verified, the following properties are satisfied

(I) $\langle p, q\rangle=S(p \bar{q})=S(q \bar{p})=\langle q, p\rangle$,

(II) $\langle p, q+r\rangle=S(p \overline{(q+r)})=S(p \bar{q}+p \bar{r})=\langle p, q\rangle+\langle p, r\rangle$,

(III) $\alpha\langle p, q\rangle=S((\alpha p) \bar{q})=\langle\alpha p, q\rangle=S(p \overline{(\alpha q)})=\langle p, \alpha q\rangle, \lambda \in R$

(IV) $\langle q, q\rangle=S(q \bar{q})=a^{2}-b^{2}-c^{2}-d^{2}$.

Thus the inner product defined here is a symmetric bilinear form but is not positive definite. The inner product defines the norm of $q=a+b i+c j+d k \in K$ as follows

$$
N(q)=\langle q, q\rangle=q \bar{q}=a^{2}-b^{2}-c^{2}-d^{2} .
$$

The norm is real-valued function and the norm of a hyperbolic quaternions satisfies the properties $N(\bar{q})=N(q)$. But $N(p q) \neq N(p) N(q)$. Let $p=p^{\alpha} e_{\alpha}, q=q^{\alpha} e_{\alpha}$ be hyperbolic quaternions where $e_{\alpha} \in\{1, i, j, k\}$. Then the relations:

$$
\langle p, q\rangle=S(p \bar{q})=\eta_{\alpha \beta} p^{\alpha} q^{\beta}
$$

defines metric $\eta_{\alpha \beta}$. To obtain its components explicitly, we choose $p=e_{\alpha}, q=e_{\beta}$ (for particular $\alpha, \beta$ ). Then

$$
p=\delta^{\alpha \mu} e_{\mu}, q=\delta^{\beta v} e_{v}
$$

where $\delta^{\alpha \beta}$ is kronecker delta. That is

$$
p^{\mu}=\delta^{\alpha \mu}, q^{v}=\delta^{\beta v}
$$

therefore

$$
\langle p, q\rangle=\frac{1}{2}\left[e_{\alpha} \overline{e_{\beta}}+e_{\beta} \overline{e_{\alpha}}\right]
$$


also

$$
\langle p, q\rangle=\eta_{\mu \nu} \delta^{\alpha \mu} \delta^{\beta v}=\eta_{\alpha \beta}
$$

and so

$$
\eta_{\alpha \beta}=\frac{1}{2}\left[e_{\alpha} \overline{e_{\beta}}+e_{\beta} \overline{e_{\alpha}}\right]
$$

giving, after a short calculation, we reach

$$
\eta_{\alpha \beta}=\left(\begin{array}{rrrr}
1 & 0 & 0 & 0 \\
0 & -1 & 0 & 0 \\
0 & 0 & -1 & 0 \\
0 & 0 & 0 & -1
\end{array}\right)
$$

which is the usual flat-space metric of Minkowski space.

\section{Euler's and De Moivre's Formulas for a Hyperbolic Quaternions}

In this section, we express Euler's formula and De Moivre's formula for hyperbolic quaternions and examine roots of hyperbolic quaternion with respect to the norm of the hyperbolic quaternions.

Every hyperbolic quaternion $q=a+b i+c j+d k(N(q)>0)$ can be written in the form

$$
q=\sqrt{N(q)}(\cosh \phi+w \sinh \phi)
$$

where $\cosh \phi=\frac{|a|}{\sqrt{N(q)}}, \sinh \phi=\frac{\sqrt{b^{2}+c^{2}+d^{2}}}{\sqrt{N(q)}}, w=\frac{1}{\sqrt{b^{2}+c^{2}+d^{2}}}(b i+c j+d k)$ is unit hyperbolic quaternion and $w^{2}=w w=1$. Since $w^{2}=1$, we have

$$
e^{w \phi}=\left(1+\frac{\phi^{2}}{2 !}+\frac{\phi^{4}}{4 !}+\ldots\right)+w\left(\phi+\frac{\phi^{3}}{3 !}+\frac{\phi^{5}}{5 !}+\ldots\right)=\cosh \phi+w \sinh \phi
$$

Moreover, this can be shown using another method. In following manner

$$
\begin{array}{r}
q=(\cosh \phi+w \sinh \phi) \Rightarrow d q=(\sinh \phi+w \cosh \phi) d \phi \\
\Rightarrow d q=w(\cosh \phi+w \sinh \phi) d \phi=w q d \phi .
\end{array}
$$

Thus, we get

$$
\int \frac{d q}{q}=\int w d \phi \Rightarrow \ln q=w \phi \Rightarrow q=e^{w \phi}=(\cosh \phi+w \sinh \phi) .
$$

Now let's prove De Moivre's formula for hyperbolic quaternion.

Theorem 3.1. Let $q=\sqrt{N(q)}(\cosh \phi+w \sinh \phi)$, where $\phi$ is a real number and $w^{2}=1$. Then

$$
q^{n}=\left(\sqrt{N_{q}}\right)^{n}(\cosh \phi+w \sinh \phi)^{n} \quad=\left(\sqrt{N_{q}}\right)^{n}(\cosh (n \phi)+w \sinh (n \phi))
$$

for every integer $n$.

Proof. We use induction on positive integers $n$. Assume that

$$
q^{n}=\left(\sqrt{N_{q}}\right)^{n}(\cosh (n \phi)+w \sinh (n \phi))
$$

holds. Then,

$$
\begin{aligned}
q^{n+1} & =\left(\sqrt{\left|N_{q}\right|}\right)^{n}(\cosh (n \phi)+w \sinh (n \phi))\left(\sqrt{\left|N_{q}\right|}\right)(\cosh \phi+w \sinh \phi) \\
& =\left(\sqrt{\left|N_{q}\right|}\right)^{n+1}\left[\begin{array}{c}
(\cosh (n \phi) \cosh \phi+\sinh (n \phi) \sinh \phi) \\
+w(\cosh (n \phi) \sinh \phi+\sinh (n \phi) \cosh \phi)
\end{array}\right] \\
& =\left(\sqrt{\left|N_{q}\right|}\right)^{n+1}[(\cosh (\phi(n+1)))+w(\sinh (\phi(n+1)))] .
\end{aligned}
$$

Hence, the formula is true. Moreover, since

$$
\begin{aligned}
q^{-1} & =\left(\sqrt{\left|N_{q}\right|}\right)^{-1}(\cosh \phi-w \sinh \phi) \\
q^{-n} & =\left(\sqrt{\left|N_{q}\right|}\right)^{-n}(\cosh (n \phi)-w \sinh (n \phi)) \\
= & \left(\sqrt{\left|N_{q}\right|}\right)^{-n}(\cosh (-n \phi)+w \sinh (-n \phi)),
\end{aligned}
$$

the formula holds for all integer.

If the power series definition

$$
\begin{aligned}
& \cosh x=1+\frac{x^{2}}{2 !}+\frac{x^{4}}{4 !}+\ldots \\
& \sinh x=x+\frac{x^{3}}{3 !}+\frac{x^{5}}{5 !}+\ldots
\end{aligned}
$$


is used for hyperbolic quaternion $w$, then we obtain

$$
\cosh w=\cos I
$$

$\sinh w=-w I \sin I$

where $I$ is complex operator.

Every hyperbolic quaternion $q=a+b i+c j+d k,(N(q)<0)$ can be written in the form

$$
q=\sqrt{|N(q)|}(\sinh \phi+w \cosh \phi)
$$

where $\sinh \phi=\frac{a}{\sqrt{|N(q)|}}, \cosh \phi=\frac{1}{\sqrt{|N(q)|}} \sqrt{b^{2}+c^{2}+d^{2}}$ and $w=\frac{1}{\sqrt{b^{2}+c^{2}+d^{2}}}(b i+c j+d k)$ is unit hyperbolic quaternion.

Theorem 3.2. Let $q=\sqrt{|N(q)|}(\sinh \phi+w \cosh \phi),(N(q)<0)$ be hyperbolic quaternion. Then

$$
q^{n}= \begin{cases}(\sqrt{|N(q)|})^{n}(\sinh n \phi+w \cosh n \phi), & n \text { is odd } \\ (\sqrt{|N(q)|})^{n}(\cosh n \phi+w \sinh n \phi), & n \text { is even } .\end{cases}
$$

The proof can be bypassed since it can be proved in same manner of the proof of the Theorem 3.1.

\subsection{The roots of a Hyperbolic Quaternions}

Theorem 3.3. Let $p=\sqrt{N(p)}(\cosh \phi+w \sinh \phi)$. Then the equation $q^{n}=p$ has only one root:

$$
q=\sqrt[2 n]{N(p)}\left(\cosh \left(\frac{\phi}{n}\right)+w \sinh \left(\frac{\phi}{n}\right)\right)
$$

in the hyperbolic quaternions which $N(q)>0$.

Proof. Assume that $q=\sqrt{N(q)}(\cosh x+w \sinh x)$ is a root of the equation $q^{n}=p$. From theorem 3.1, we have $q^{n}=(\sqrt{N(q)})^{n}(\cosh (n x)+w \sinh (n x))$ . Thus, $x=\frac{\phi}{n}$ and $|N(q)|=\sqrt[n]{|N(p)|}$. Then, $q=\sqrt[2 n]{N(p)}\left(\cosh \left(\frac{\phi}{n}\right)+w \sinh \left(\frac{\phi}{n}\right)\right)$ is a root of the equation. If we suppose that there are two roots satisfying the equality, we obtain that these roots must be equal to each other.

Example 3.4. We find the roots of the equation $q^{2}=\sqrt{3}+i+j$. Here $p=\sqrt{3}+i+j$ is a hyperbolic quaternion such that $N_{p}=1$. Then, $p$ can be written as

$$
p=\cosh (\ln (\sqrt{3}+\sqrt{2}))+w \sinh (\ln (\sqrt{3}+\sqrt{2}))
$$

where $w=\frac{1}{\sqrt{2}}(i+j)$. From theorem 3.3, the root of the equation

$$
q^{2}=\cosh (\ln (\sqrt{3}+\sqrt{2}))+w \sinh (\ln (\sqrt{3}+\sqrt{2}))
$$

is as follows

$$
q=\cosh \left(\frac{\ln (\sqrt{3}+\sqrt{2})}{2}\right)+w \sinh \left(\frac{\ln (\sqrt{3}+\sqrt{2})}{2}\right) .
$$

Theorem 3.5. Let $p=\sqrt{|N(p)|}(\sinh \phi+w \cosh \phi)$ be hyperbolic quaternion. Then the solution of hyperbolic quaternion $q^{n}=p$ 1. doesn't exist if $n$ is an even number,

2. has only one root $q=\sqrt[2 n]{|N(p)|}\left(\sinh \frac{\phi}{n}+w \cosh \frac{\phi}{n}\right)$ if $n$ is an odd number in the hyperbolic quaternions which $N(q)<0$,

Proof. If $n$ is an even number, the norm of the $n^{\text {th }}$ power of hyperbolic quaternion will be positive and in this case there is no solution. So, let $q=\sqrt{|N(q)|}(\sinh x+w \cosh x)$ be root of the equation $q^{n}=p$ such that $n$ is an odd number. Then

$$
q^{n}=\sqrt{|N(q)|^{n}}(\sinh n x+\cosh n x)=\sqrt{|N(p)|}(\sinh \phi+\cosh \phi)
$$

and we get $x=\frac{\phi}{n}$ and $|N(q)|=\sqrt[n]{|N(p)|}$. 
Example 3.6. We find the roots of the equation $q^{3}=1+\sqrt{2} j$. Here $p=1+\sqrt{2} j$ is a hyperbolic quaternion such that $N(p)=1$. Then $p$ can be written as

$$
p=\sinh (\ln (1+\sqrt{2}))+w \cosh (\ln (1+\sqrt{2}))
$$

where $w=j$. From theorem 3.4 the root of the equation

$$
q^{3}=\sinh (\ln (1+\sqrt{2}))+w \cosh (\ln (1+\sqrt{2}))
$$

is

$$
q=\sinh \left(\frac{\ln (1+\sqrt{2})}{3}\right)+w \cosh \left(\frac{\ln (1+\sqrt{2})}{3}\right)
$$

\section{References}

[1] Hamilton, W.R., Elements of Quaternions, London Longmans Green, 1866

[2] Cho, E., De Moivre's Formula for Quaternions, Appl. Math. Lett. 6 (1998), 33-35.

[3] Özdemir, M., The Roots of a Split Quaternion, Appl. Math. Lett. 22 (2009), 258-263.

[4] MacFarlane, A., Hyperbolic Quaternions, Proc. Roy. Soc. Edinburgh, 1900, pp. 169-181.

[5] Ward, JP., Quaternions and Cayley Numbers Algebra and Applications, Boston(SPA), Kluwer Academic, 1997.

[6] Demir, S., Tanışl1, M., Candemir, N., Hyperbolic Quaternions Formulation of Electromacnetism, Adv. Appl. Clifford Algebras, 20 (2010), $547-563$.

[7] Niven I., The Roots of a Quaternion, Amer. Math. Monthly 449 (6) (1942) 386-388.

[8] Brand L., The Roots of a Quaternion, Amer. Math. Monthly 49 (8) (1942) 519-520. 